\title{
Bone turnover markers: response to comments by Seeman and Nguyen
}

\author{
K. E. Naylor ${ }^{1}$ - R. M. Jacques ${ }^{2}$ - M. Paggiosi ${ }^{1}$ - F. Gossiel ${ }^{1}$ • N. F. Peel ${ }^{1}$ \\ E. V. McCloskey ${ }^{1}$ • J. S. Walsh ${ }^{1} \cdot$ R. Eastell ${ }^{1}$
}

Received: 21 October 2015 / Accepted: 25 October 2015 / Published online: 11 November 2015

(C) International Osteoporosis Foundation and National Osteoporosis Foundation 2015

\section{Dear Editor,}

We read with interest the various comments from Seeman and Nguyen [1]. Many of these are speculative interpretations of data about differences in bisphosphonates that may or may not be of clinical relevance. While of interest, we feel that the comments they make are in the most part irrelevant to our article [2]. However, they did raise a couple of issues that we would like to clarify.

We did not report the analysis of covariance as the purpose of our article was not to compare treatments, but rather to examine the definition and detection of responses to treatment in individuals. As we described, the response rates defined by bone turnover markers (BTM) were somewhat lower in the women receiving risedronate. This was also confirmed in an analysis of covariance; this mixed model ITT analysis did indeed use logtransformed data with baseline BTM as co-factor and ibandronate as the comparator for treatment effect. There remained a significant treatment effect for CTX and NTX $(P<0.001)$; (the risedronate group was different to ibandronate whereas alendronate was not). Baseline BTM was significant for all BTM (BAP $P=0.001$, OC $P=0.007$, PINP, CTX and NTX, all $P<0.001)$ in that those with higher baseline values experienced significantly greater decreases in the individual BTM.

R. Eastell

r.eastell@sheffield.ac.uk

1 Academic Unit of Bone Metabolism, Mellanby Centre for Bone Research, Metabolic Bone Centre, Northern General Hospital, Herries Road, Sheffield, South Yorkshire S5 7 AU, UK

2 School of Health and Related Research, University of Sheffield, Sheffield, UK
The correspondents misunderstand the origin of the terms 'resorption' and 'formation' markers. We don't have to assume that osteoclasts resorb bone and release collagen fragments or that osteoblasts secrete proteins such as osteocalcin. We know from in vitro studies that bone turnover markers are products of the work of bone cells [3]. They are not in any way surrogates of cellular activity; indeed, Seeman and Nguyen interpret the changes in BTM during early treatment in terms of the impact of treatment on cellular changes. However, we do not propose in our paper that we are able to use BTM to replace bone histomorphometry, nor that we can infer from the changes in their levels whether these changes are occurring in a particular bone envelope. We agree that it is appropriate to evaluate (but not define) bone turnover markers for their associations with bone turnover rates using radiotracer kinetics [4] or bone histomorphometry [5].

\section{References}

1. Seeman E, Nguyen TV (2015) Bone remodeling markers: so easy to measure, so difficult to interpret. Osteoporos Int. doi:10.1007/ s00198-015-3374-9

2. Naylor KE, Jacques RM, Paggiosi M, Gossiel F, Peel NF, McCloskey EV, Walsh JS, Eastell R. Response of bone turnover markers to three oral bisphosphonate therapies in postmenopausal osteoporosis: the TRIO study. Osteoporos Int. doi:10.1007/s00198-015-3145-7

3. Szulc P (2012) The role of bone turnover markers in monitoring treatment in postmenopausal osteoporosis. Clin Biochem 45(12):907-919

4. Eastell R, Colwell A, Hampton L, Reeve J (1997) Biochemical markers of bone resorption compared with estimates of bone resorption from radiotracer kinetic studies in osteoporosis. J Bone Miner Res 12(1):59-65

5. Eriksen EF, Hodgson SF, Eastell R, Cedel SL, O'Fallon WM, Riggs BL (1990) Cancellous bone remodeling in type I (postmenopausal) osteoporosis: quantitative assessment of rates of formation, resorption, and bone loss at tissue and cellular levels. J Bone Miner Res 5(4):311-319 